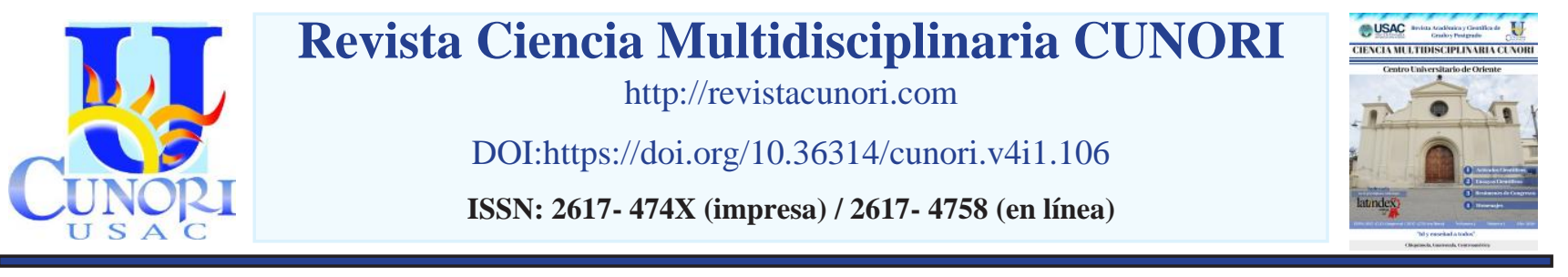

Como citar el artículo

Villela, C., Locón, A. y Gatica S. (2020). Interculturalidad la competencia del futuro Médico y Cirujano. Revista Ciencia Multidisciplinaria CUNORI 4(1). 21-28. DOI: https://doi.org/10.36314/cunori.v4i1.106

\title{
Interculturalidad la competencia del futuro Médico y Cirujano
}

\section{Intercultural competence of the future Doctor and Surgeon}

\author{
Claudia Esmeralda Villela, Agustín locón y Sergio Gatica \\ Centro Universitario de Oriente (CUNORI, Universidad de San Carlos de Guatemala \\ Recibido: 12 de noviembre de 2019 / Revisión: 14 de enero de 2020/ Aceptado: 20 de mayo de 2020 \\ Disponible en internet el 29 de mayo de 2020 \\ *Autor para correspondencia. \\ Correo electrónico: villelaclaudiaesmeralda@gmail.com
}

\section{Resumen}

\begin{abstract}
【 os espacio reales de interacción en la formación universitaria pública, pueden no representar en buena medida la construcción de la interculturalidad, existe sin ninguna duda un sector significativo de profesionales, dentro y fuera del campo estrictamente académico, que aunque dispersos luchan y aportan permanentemente, desde la cátedra y la investigación, para la construcción del Estado intercultural al que debe aspirar principalmente una sociedad como Guatemala. Ese plurilingüismo, multiculturalidad y multietnicidad, hace que sea un país sumamente rico. Y fortalece la idea de que la educación debe tener un componente plurilingüe y multicultural. El currículo base de la escuela primaria y secundaria enfoca esta situación de manera pertinente, el punto de vista multicultural y la práctica de la interculturalidad son fundamentales, en educación superior debería ser más, por cuanto que la inequidad en estos niveles se expresa con toda la plenitud. Para dar respuesta a la pregunta ¿Incluye la formación intercultural el pensum de la carrera Médico y Cirujano del Centro Universitario CUNORI? Se revisó las leyes y reglamentos, la malla curricular, de la Universidad de San Carlos, el pensum y guías programáticas. De manera general en la malla curricular y las leyes y reglamentos establecen el eje intercultural, el pensum y guías programáticas no la incluyen. Es necesario integrar al pensum de la carrera la educación intercultural desde tres perspectivas: Desde el respeto a las costumbres, el bilingüismo y medicina natural como tratamiento terapéutico, para que los futuros profesionales de la salud conozcan las costumbres de los pacientes y formen una visión intercultural de la salud de los pueblos de acuerdo a sus creencias.
\end{abstract}

Palabras clave: educación, intercultural, médico y cirujano.

\section{Abstract}

The real spaces of interaction in public university education, may not represent to a large extent the construction of interculturality, there is undoubtedly a significant sector of professionals, inside and outside the strictly academic field, who although dispersed fight and contribute permanently, from the chair and research, for the construction of the intercultural State to which a society like Guatemala must aspire mainly. This multilingualism, multiculturalism and multiethnicity makes it an extremely rich country. And it strengthens the idea that education must have a multilingual and multicultural component. The basic curriculum of primary and secondary school approaches this situation in a pertinent way, the multicultural approach and the practice of interculturality are fundamental, in higher education it should be more, since the inequity at these levels is fully expressed.To answer the question, does intercultural training include the curriculum of the Medical and Surgeon degree at the CUNORI University Center? The laws and regulations, the curricular mesh of the University of San Carlos, the curriculum and program guides were reviewed. In general, in the curricular grid and the laws and regulations establish the intercultural axis, the curriculum and program guides do not include it. It is necessary to integrate intercultural education into the career curriculum from three perspectives: From respect 
for customs, bilingualism and natural medicine as therapeutic treatment, so that future health professionals know the customs of patients and form an intercultural vision of people's health according to their beliefs.

Keywords: interculturality, intercultural higher education, intercultural health, bilingualism

\section{Introducción}

El plurilingüismo, multiculturalidad y multietnicidad, hace que Guatemala sea un país sumamente intercultural, compréndase la palabra "intercultural" como el proceso de comunicación e interacción entre personas con identidades culturales específicas, esto incluye que si reside, estudia o trabaja en la región oriental de Guatemala, en los departamentos de Jutiapa, Zacapa, Chiquimula y Jalapa, debe conocer la cultura y el idioma de por lo menos las etnias Chortí, Pocoman y Xincas.

La educación en todos los niveles, debe tener un componente plurilingüe y multicultural. De acuerdo a las demandas sociales, éstas deben vincular el currículo a la realidad nacional en los distintos ámbitos. Esto significa que los programas de estudios de la formación de profesionales en todas sus carreras deben incluir como eje transversal la educación intercultural, especialmente en las carreras social-humanística como la carrera Médico y Cirujano.

Los planes y programas de la carrera en mención en su mayoría responden a la tradicional educación occidental. Ha representado, en buena medida, la necesidad de preparar recurso humano para trabajar en contextos interculturales. Sin embargo lamentablemente la educación bilingüe intercultural no está establecida en el pensum de estudios de la carrera de Médico y Cirujano, se lee el requisito de estudio de 18 meses del Idioma Extranjero Inglés, como cursos obligatorios, lastimosamente los médicos egresados del CUNORI, trabajarán en la región Chortí, sin poseer conocimientos de interculturalidad, ni aprendizaje del idioma.

Desde este enfoque de formación se inicia con la sensibilidad y el respeto a la diversidad. Sin embargo no solamente este enfoque se debe tomar en cuenta; la universidad también los debe formar a los futuros profesionales especialmente de la carrera de médico y cirujano, quienes serán los que atenderán a los pacientes en las comunidades para que conozcan desde las aulas el tema intercultural en lo que respecta a la diversidad cultural de los pacientes, desde la cosmovisión de los pueblos.

\section{Materiales y métodos}

La investigación es descriptiva a través de documentos de fuentes primarias y secundarias, se revisó leyes y reglamentos de la Universidad de San Carlos, la malla curricular, el pensum y las guías programáticas de la carrera Médico y Cirujano. Se citó autores expertos en el tema interculturalidad como Chúa, Iglesias, Mato, Leyes y reglamentos de la Universidad de San Carlos, Informes del Ministerio de Salud Pública y Asistencia Social, informes del Banco Interamericano de Desarrollo y el Diccionario de la Real Academia de la Lengua Española. 


\section{Resultados}

\section{La interculturalidad un desafíos de la educación superior en Guatemala}

En Guatemala desde la firma de los acuerdos de paz del 29 de diciembre de 1996, el sistema educativo superior guatemalteco está comprometido a impulsar una reforma, con base en el acuerdo sobre Identidad y Derechos de los Pueblos Indígenas, en ese sentido desde el año 1997 la única universidad estatal, autónoma y con personalidad jurídica, establece los objetivos principales fijados en la reforma universitaria; son los de proveer una sólida formación técnica, científica y humanista; fortalecer y desarrollar los valores, las actitudes del pluralismo y respeto a la vida y los derechos humanos, formadora de actitudes críticas, creativas, propositivas y de sensibilidad social. Con estos objetivos, lo que pretenden es establecer un sistema educativo superior universitario intercultural y multilingüe.

Como afirma Chuá (2014), es necesario en primer lugar aproximarse a la comprensión de lo que entendemos por "cultura" y luego la posibilidad de que, de aquellos contenidos tipificados como tal, podamos emprender el camino hacia un hecho o fenómeno de interculturalidad.

El Diccionario de la Real Academia de la Lengua conceptualiza la cultura como "Resultado de cultivar los conocimientos humanos", agrega otra significación: "Las costumbres, conocimientos y grado de desarrollo artístico, científico, industrial, en una época o grupo determinado" y añade Cultura Popular como "Conjunto de manifestaciones en que se expresa la vida tradicional de un pueblo". Chúa cita a Olmedo España, "la cultura es el norte que deben pensar las universidades porque caracteriza el estilo de vida de una región". La interculturalidad se manifiesta en la diversidad, en la construcción armónica de la convivencia y en la solución sin violencia de todo conflicto surgido por razones de origen cultural.

Por lo tanto la "cultura" y la "interculturalidad" se manifiestan y construyen como proceso de vida y no necesariamente porque se incentive o diseñe desde la escolarización. Razón para pensar que, si ese fuese el deseo, debería de hacerse con toda la coherencia posible en todas las etapas de la educación escolarizada y no solo en el nivel universitario. Lo importante de notar es que es casi imposible pensar que un proceso de educación de vida que ha transcurrido, con afirmación de la "monoculturalidad" desde el nacimiento de toda la inteligencia viviente en su núcleo familiar y reafirmado en un sistema educativo formal, fragmentado y anárquico, pueda ser corregido absolutamente en el último escalón de la educación formal. Quizá las mismas resistencias a la creación de "instituciones interculturales como tal" o de los programas o actividades puntuales que tratan de responder a ese concepto en instituciones de educación universitaria "tradicionales".

En este espacio se presenta solamente a la Universidad estatal de Guatemala, por ser la única universidad pública del país, ¿Qué ha hecho en cuanto a la construcción de la interculturalidad? Dadas las características no constituye un espacio diseñado como una institución intercultural de origen, es decir es un espacio de educación superior, a decir Mato (2011), "convencional". Por lo que vale la pena citar a Chúa (2014) al refererirse que existen actividades programáticas, con reconocimiento de la multiculturalidad y pluriculturalidad pero más como referencias teóricas y dispersas en los distintos niveles de distintas facultades, no responden a una política institucional, como una universidad en su totalidad, de construcción de interculturalidad. 
Si en la educación primaria y secundaria de Guatemala el enfoque multicultural y la práctica de la interculturalidad son fundamentales, en educación superior debería ser más, por cuanto que la inequidad en estos niveles se expresa con toda la plenitud. El otro aspecto de la interculturalidad en la universidad se refiere a la interacción de los estudiantes indígenas con los no indígenas. Es decir, ya losestudiantes habiendo logrado ingresar a la Universidad, ¿cómo es el comportamiento interrelacional con los otros estudiantes, con las autoridades, con los maestros y maestras, con el personal de la administración educativa?

La sola posesión de la indumentaria indígena en los estudiantes, conlleva ya un tipo especial de relación con los demás. Puede ser que se niegue la discriminación, pero lo que sí se observa es que de algún modo se le señala a la mujer indígena por su indumentaria, por su manera de comportarse, y casi se le pide que se vista a lo "occidental" y que cambie sus actitudes.

En la Facultad de Ciencias Médicas y en Enfermería del campus central, por ejemplo, si no se prohíbe explícitamente, sí se les indica a las mujeres estudiantes que se pongan "el uniforme blanco" o la bata blanca porque es más apropiada esta ropa que la indumentaria que tienen puesta ¿Es igual el trato hacia las estudiantes indígenas que hacia las no indígenas por parte de profesores o incluso personal administrativo? Creo que no, que existen formas sutiles de discriminación, aunque la mentalidad liberal del universitario trate de convencer de lo contrario. Y que estas formas sutiles de discriminación no contemplan únicamente el vestido, sino que también la lengua, el apellido, las costumbres, la manera de insertarse en el mundo y la manera de percibirlo. Como señaló un estudiante indígena de medicina: "Aquí entramos indígenas pero luego-luego nos hacen ladinos, aprendemos de los ladinos y con los ladinos, salimos como médicos ladinos y hasta aprendemos a cobrar como ladinos”.

En un país como Guatemala, profundamente multicultural, plurietnico y multilingüe, la interculturalidad en educación superior es indispensable. Siendo la Universidad de San Carlos la única del Estado existente del país, debe tener toda una conceptualización sobre el tema y debe emprender políticas públicas para sostener la equidad y para favorecer un desarrollo integral para los individuos y la sociedad. (Íbidem). Desde este enfoque de formación se inicia con la sensibilidad y el respeto a la diversidad. Sin embargo no solamente este enfoque se debe tomar en cuenta, la universidad también los debe formar a los futuros profesionales especialmente de la carrera de médico y cirujano, quienes serán los que atenderán a los pacientes en las comunidades para que conozcan desde las aulas el tema de intercultural en lo que respecta a la diversidad cultural de los pacientes, desde la cosmovisión de los pueblos.

\section{Las competencias interculturales de los futuros médicos y cirujanos}

Según Iglesias (2015), las competencias interculturales hoy en día son un elemento clave para la comprensión de la interculturalidad ya que permiten identificar condiciones facilitadoras o dificultadoras para el complejo marco de interacción de un contexto multicultural. También, porque las posibilidades para desenvolverse socialmente o educativamente en un nuevo contexto cultural se encuentran en relación al desarrollo de las competencias interculturales, que se fundamentan desde habilidades y destrezas de base cognitiva, afectiva o comportamental. 
La educación bilingüe intercultural no está establecida en el pensum de estudios en la carrera de medicina, solo está el estudio de 18 meses del Idioma Extranjero Inglés, como cursos obligatorios como requisito de graduación. El aprendizaje del idioma maya de la región no se le da importancia al no aparecer en el pensum de la carrera.

\section{La interculturalidad en el currículum en la carrera de medicina}

Al no integrarse la educación bilingüe intercultural y el conocimiento de cultura, salud intercultural correspondiente a la región, en la carrera médico y cirujano, los conocimientos intercultural en general que tienen los estudiantes, estarán lejos de lograr la visión y misión y de cumplir con las políticas con el enfoque de interculturalidad como lo establece el plan estratégico de la universidad estatal para el año 2022. No solo integrar temas de salud intercultural si no también el bilingüismo. Con estos temas lo que se propone es sensibilizar a los futuros profesionales de la medicina, formarlos desde que son estudiantes, para que al graduarse puedan desempeñarse con respeto a las situaciones de costumbres que presenten los pacientes. Lo que pretenden las nuevas políticas del ministerio de salud es integrar los saberes de los sabios indígenas y los profesionales de la salud, para aliviar en forma rápida las dolencias de los pacientes. El respeto y reconocimiento de parte del personal médico para los guías espirituales, comadronas y sabios de las comunidades, generalmente analfabetas y monolingües. Si Chiquimula según los indicadores de desarrollo ocupa el segundo lugar a nivel nacional de población en extrema pobreza. Así como está la crisis hospitalaria sin recursos en los hospitales, el médico le da a los pacientes una receta que no puede comprar la medicina en la farmacia, pudiendo recetar plantas medicinales para la cura de las enfermedades en el primer nivel de atención, pero como no ha sido formado no va a recetar la medicina natural.

Es necesario integrar al pensum de la carrera la educación intercultural desde tres perspectivas: desde el respeto a las costumbres, el bilingüismo y medicina natural como tratamiento terapéutico. Lo que se pretende con la integración, que los futuros profesionales respeten, comprendan y toleren, por el solo hecho de ser el paciente una persona. Este respeto incluye las creencias y culturas diversas. Evidentemente, los límites de ese respeto, individual o colectivo, se trazan donde en consideraciones de igualdad, el otro violenta la dignidad.

Es necesario resaltar que el respeto, del trabajador de la salud, no significa que deba aceptar como bueno todo lo que dice el paciente, sean estas cristianas, mayas, musulmanas entre otras. Desde la perspectiva de su trabajo, lo importante es reconocer la obligación de conservar una actitud de escucha y comunicación adecuada, lo cual contribuye a comprender y aprender de las personas.

Las normas con pertinencia cultural del sistema de salud indígena comprende el conjunto de actores, elementos terapéuticos, organización, coordinación, relación y modelo; que sustenta y le da las características esenciales. Su presencia en la cultura de los pueblos indígenas es genuina, legítima y dinámica; constituyen los mejores sistemas para la prevención, atención y restablecimiento de su salud, por ser parte de su cultura y comprende sus formas de vida y cosmopercepción. (Ministerio de Salud Pública y Asistencia Social, Acuerdo Ministerial 1632-2009). 
En los servicios salud con pertinencia cultural evidencia respeto a los elementos sagrados del sistema de salud de pueblos indígenas, a los valores y cosmovisión que desde tiempo milenarios los ha estudiado la ciencia, determinando que son efectivos en la curación de enfermedades.

\section{Los cuatro pueblos de Guatemala}

Según la Información sobre los Pueblos Indígenas de Guatemala como insumo para el Proyecto Regional de Manejo Integrado de Ecosistemas por Pueblos Indígenas y Comunidades de Centroamérica Cooperación Técnica BID (2003). Guatemala está integrada por cuatro pueblos distintos, el Pueblo Maya, Pueblo Garífuna, Pueblo Xinka y Pueblo Ladino, la UNESCO también los reconoce como pueblos. El Pueblo Maya, está compuesto por 22 comunidades lingüísticas, Achi', Akateko, Awakateko, Ch'orti', Chuj, Ixil, Itza', Chalchiteka Kaqchikel, K'iche', Mam, Mopan, Poqomam, Poqomchi', Popti', Q'anjob'al, Q'eqchi', Sakapulteko, Sipakapense, Tektiteko, Tz'utujil y Uspanteko; y los Pueblos Garífuna, Xinca y Ladino, según información publicada en la página de la Academia de las Lenguas Mayas de Guatemala.

Más del 60\% de la población guatemalteca es indígena, sufren los efectos de un régimen de discriminación por medio de la desigualdad que existe, la falta de oportunidades de educación para la población indígena, pero no es solamente en ese aspecto la discriminación, también se da en las relaciones con los ladinos, el indígena es respetuoso saluda y trata de usted a los ladinos, ellos son lo contrario tratan al indígena de vos y no lo saludan con la misma reverencia que lo hace el indígena.

\section{La formación de profesores en educación intercultural de la carrera Médico y Cirujano}

Aunque lo digan las políticas y los estatutos legales, no existe la formación intercultural en la carrera médico y cirujano. El enfoque intercultural surge según el Doctor Mato (2009) cuando se reconocen las diferencias y se evita anular o ignorar miradas, significa valorar y legitimar los pensamientos y prácticas distintas a las académicas convencionales, rompiendo con la idea de imponernos epistemológicamente a las cultura y grupos humanos definidos en la alteridad.

La educación superior debe ser con un enfoque intercultural así como lo establece el plan estratégico 2022 de la universidad estatal, dado que la sede universitaria está ubicada en la región Chortí, por lo que el personal docente y administrativos atienden estudiantes ladinos pobres, y por las cercanías con los Xincas de Jutiapa y Pocomames de Jalapa.

Cada uno de los pueblos de Guatemala tienen normas de convivencia basadas en la dignidad de la vida humana, la integridad moral y personal, la tradición y la cultura, las cuales giran en torno a la justicia, el amor y el equilibrio; la armonización en las relaciones interpersonales, por medio de la educación y el respeto, mismos que deben estar intrínsecos en el programa curricular empoderado por los docentes. A veces sucede lo contrario el estudiante se encuentra con docentes incomprensibles que no se adaptan al contexto sociocultural de los estudiantes. 
La realidad del currículum universitario es creado al estilo occidental centrado en lo urbano y convencional, al parecer como que los futuros profesionales no trabajarán en las áreas rurales o con gente pobre en las comunidades urbanas. Igualmente si la universidad forma a los docentes para la convivencia efectiva en la interculturalidad, los estudiantes quienes son los futuros profesionales que la mayoría trabajará atendiendo personas de diversas culturas, en ese sentido la formación intercultural para los estudiantes debe estar integrada como un eje transversal, por lo que es necesario que los docentes tengan el conocimiento del tema para formar a los estudiantes.

Generar desde la universidad, cambios para transformar la acción docente desde la perspectiva crítica, comprensiva desde el fortalecimiento del reconocimiento de saberes que lleven al logro de las políticas con enfoque intercultural, con el propósito que si los docentes están sensibilizados con las políticas de pertinencia intercultural sabrán transmitirlas a los estudiantes, y que a través de la educación construir el conocimiento desde la academia, que aporte sentido a un país pluricultural y diverso como lo es Guatemala.

\section{Discusión}

Concretamente el desarrollo de las competencias interculturales se relaciona con la capacidad de dar respuesta a situaciones específicas, desde la atención a elementos favorecedores o dificultadores de la propia interacción social.

Las competencias interculturales atienden y combinan aspectos cognitivos, afectivos y conativos. Orientando al desarrollo de procesos de introspección, a la compresión del grado de interacción positiva que tengan los participantes en un contexto educativo, la calidad de la relación comunicativa como indicador de comprensión de los significados culturales que se comparten. Además, los contextos educativos que son descritos como multiculturales, evidencian desde algunos de sus participantes y equipos educativos, un cierto grado de incertidumbre originado en el hecho de tener que afrontar un nuevo contexto educativo y cultural.

Es necesario integrar al pensum de la carrera la educación intercultural desde tres perspectivas: desde el respeto a las costumbres, el bilingüismo y medicina natural como tratamiento terapéutico. Para que los futuros profesionales de la salud conozcan las costumbres de los pacientes y formar una visión intercultural de la salud de los pueblos de acuerdo a sus creencias.

\section{Agradecimientos}

Se agradece a la carrera Médico y Cirujano del Centro Universitario de Oriente, por participar en el estudio. A los asesores Dr. Agustín Locón y al Maestro en Ciencias Sergio Gatica y Doctora Teresa Mosquera, por el aprendizaje en el Diplomado en Salud Intercultural. 


\section{Referencias}

Acuerdos de Paz (1996). Aspectos socioeconómicos y situación agraria. Acuerdo sobre identidad y derechos de los pueblos indígenas. Páginas 85 y 118 . Guatemala.

Chuá, C. (2014) Retos y desafíos de la educación superior actual. ¿Hay crisis? S/e. Esger: Guatemala.

Cooperación Técnica Banco Interamericano de Desarrollo -BID- (2003). Información sobre los Pueblos Indígenas de Guatemala como insumo para el Proyecto Regional de Manejo Integrado de Ecosistemas por Pueblos Indígenas y Comunidades de Centroamérica.

Iglesias, E. (2015) Educación Intercultural en el Tiempo Libre: Acción educativa en tiempos neoliberales. España: Editorial Popular.

Mato, Daniel. (2009). Educación Superior, Colaboración Intercultural y Desarrollo Sostenible/Buen Vivir. Experiencias en América Latina. Caracas: UNESCO-IESALC.

Ministerio de Salud Pública y Asistencia Social. (2009). Normas con pertinencia cultural hacia la interculturalidad. Guatemala.

Real Academia de la Lengua (1980 y 2001). Diccionario de la Lengua Española

USAC. (2003). Plan Estratégico 2022. Versión ejecutiva aprobada por el Consejo Superior Universitario en su sesión celebrada el 26 de noviembre de 2003 Punto cuarto, del acta No. 28-2003. Guatemala.

USAC. (2014). Políticas Generales de la Facultad de Ciencias Médicas. USAC. Guatemala.

USAC. (2006). Leyes y Reglamentos. Dirección de Asuntos Jurídicos. USAC. Guatemala.

\section{Sobre la autora}

\section{Claudia Esmeralda Villela}

Doctora en Educación egresada de Universidad Mariano Gálvez de Guatemala, realizó la investigación "La formación que en materia de interculturalidad reciben los estudiantes de la carrera Médico y Cirujano” en una Universidad Estatal de la cabecera departamental de Chiquimula, Guatemala”.

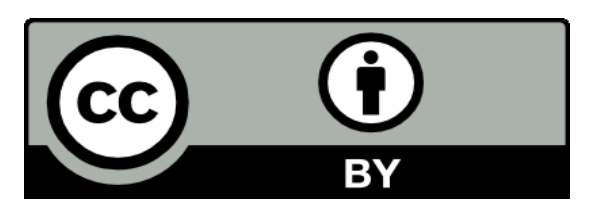

Este texto está protegido por una licencia CreativeCommons 4.0.

Usted es libre para compartir, copiar y redistribuir el material en cualquier medio o formato y adaptar el documento, remezclar, transformar y crear a partir del material para cualquier propósito, incluso comercialmente, siempre que cumpla la condición de atribución: usted debe reconocer el crédito de una obra de manera adecuada, proporcionar un enlace a la licencia, e indicar si se han realizado cambios. Puede hacerlo en cualquier forma razonable, pero no de forma tal que sugiera que tiene el apoyo del licenciante o lo recibe por el uso que hace. 